\title{
A GENERALIZATION OF TONG'S THEOREM AND PROPERTIES OF PAIRWISE PERFECTLY NORMAL SPACES
}

\author{
MANUEL LÓPEZ-PELLICER and ANGEL GUTIÉRREZ
}

(Received 5 January 1984; revised 28 June 1984)

Communicated by J. M. Rubinstein

\begin{abstract}
In this paper we give some properties of the pairwise perfectly normal spaces defined by Lane. In particular we prove that a space $(X, P, Q)$ is pairwise perfectly normal if and only if every $P(Q)$-closed set is the zero of a $P(Q)$-l.s.c. and $Q(P)$-u.s.c. function. Also we characterize the pairwise perfect normality in terms of sequences of semicontinuous functions by means of a result which contains the known Tong's characterization of perfectly normal topological spaces, whose proof we modify by using the technique of $\mathscr{E}$-binary relations.
\end{abstract}

1980 Mathematics subject classification (Amer. Math. Soc.): primary 54 E 55; secondary 54 B 10, 54 B

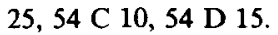

Keywords and phrases. Ginary relation, pairwise perfectly normal, perfect functions, product, quotient, semicontinuous function, subspace, sum.

\section{Introduction}

In his paper [4], Lane defines the pairwise perfectly normal bitopological spaces and proves some properties of these spaces. Later, papers of Patty [6], Arya and Singhal [1] and Romaguera [7] appeared, where are stated some properties of the pairwise perfect normality. Now we prove two characterizations and other properties, some of them in relation with important types of functions. We denote the upper (lower) semicontinuous functions as u.s.c. (1.s.c.) functions.

In the second section we characterize the pairwise perfectly normal spaces $(X, P, Q)$ as those in which every $P(Q)$-open set is a cozero set. Also we give a

(ㄷ) 1985 Australian Mathematical Society $0263-6115 / 85 \$ A 2.00+0.00$ 
bitopological form of a characterization of perfectly normal topological spaces of Tong [8, Theorem 3]; in this proof we use a technique based on the $\mathscr{C}$-binary relations defined in [5]; from the more general proof of the bitopological statement we can obtain easily a topological one which modifies the original Tong's topological proof of the theorem.

The third section is devoted to the study of the relations among the pairwise perfect normality and the functions, the bitopological product and sum.

\section{Characterizations of pairwise perfectly normal spaces}

2.1. Definition $[4,2.14]$. A bitopological space $(X, P, Q)$ is pairwise perfectly normal if it is pairwise normal and every $P(Q)$-closed subset of $X$ is $G_{\partial}$ with respect to $Q(P)$.

2.2. TheOREM. A space $(X, P, Q)$ is pairwise perfectly normal if and only if it satisfies

(a) for every P-closed set $A \subset X$ there is a P-l.s.c. and Q-u.s.c. function $f_{A}$ : $X \rightarrow[0,1]$ such that $f_{A}^{-1}(0) \cdot=A$,

(b) for every $Q$-closed set $B \subset X$ there is a Q-l.s.c. and P-u.s.c. function $g_{B}$ : $X \rightarrow[0,1]$ such that $g_{B}^{-1}(0)=B$.

Proof. We prove the necessity by using the technique of Urysohn's lemma. As $X$ is pairwise perfectly normal, given a $P$-closed set $A \subset X$, we may write $A=\bigcap_{n=1}^{\infty} V_{n}, V_{n} Q$-open and satisfying $V_{n+1} \subset V_{n}$ for every $n$.

By the pairwise normality of $X$ and the classical technique, we obtain the family $\{Q(\alpha), \alpha \in D\}$, where $D$ is the set of the dyadic numbers in $[0,1]$, of subsets of $X$ such that $Q(0)=\varnothing$ and $Q(1)=X, A \subset Q(\alpha) \subset V_{1}$ for every $\alpha \in D-\{0,1\}, P-\operatorname{cl}(Q(\alpha)) \subset Q-\operatorname{int}(Q(\beta))$ when $\alpha<\beta$, and $Q\left(1 / 2^{n}\right) \subset V_{n+1}$ for every natural $n$.

Then $\{Q(\alpha), \alpha \in D\}$ defines the $P$-1.s.c. and $Q$-u.s.c. function $f_{A}: X \rightarrow[0,1]$ such that $f_{A}(x)=\inf \{\alpha \in D: x \in Q(\alpha)\}$. We have $f_{A}^{-1}(0)=A$ because $\cap\{Q(\alpha)$ : $\alpha \in D-\{0\}\}=A$.

Similarly we obtain the function $g_{B}$ for $(b)$.

The sufficiency is immediate.

2.3. CoRollary. A space $(X, P, Q)$ is pairwise perfectly normal if and only if for every $P(Q)$-open set $A \subset X$ there is a $P(Q)$-l.s.c. and $Q(P)$-u.s.c. function $f$ : $X \rightarrow[0,1]$ such that $A$ is the cozero set of $f$. 
The last corollary is the same as the result stated by Lane [4] on page 247; however we note that the concept of $Q$-zero set with respect to $P$ introduced by Lane $[4,2.7]$ does not coincide with the usual concept of zero set, that we use here.

Later we shall prove the characterization which extends Tong's theorem. We use the techniques of the $\mathscr{b}$-binary relations introduced in [5]. In order to help with reading, we include the necessary definitions and statement. We denote by $P(X)$ the family of subsets of $X$ and $\mathscr{C}$ is a subset of $P(X)$ stable for countable unions. Moreover, $Q=\left\{q_{n}\right\}_{n=1}^{\infty}$ is the set of the rational numbers.

2.4. Definition [5]. Let $X$ be a set, $\mathscr{C} \subset P(X)$ and let $\rho$ be a binary relation on $P(X)$. We say that $\rho$ is a $\mathscr{C}$-binary relation if

(a) given two finite subsets $\mathscr{A}, \mathscr{B} \subset P(X)$, such that $A \rho B$ for every $A \in \mathscr{A}$ and $B \in \mathscr{B}$, always there is a $C \in \mathscr{C}$ such that $A \rho C$ and $C \rho B$ for every $A \in \mathscr{A}$ and $B \in \mathscr{B}$.

(b) if $D \rho E, F \subset D$ and $E \subset G$, then $D \subset E$ and $F \rho G$.

2.5. LeMMA [5, 2]. Let $\rho$ be a Cbinary relation on $P(X)$. Let $\left\{F\left(q_{n}\right): q_{n} \in Q\right\}$ and $\left\{G\left(q_{n}\right): q_{n} \in Q\right\}$ be families of subsets of $X$ such that $F\left(q_{m}\right) \subset F\left(q_{n}\right)$, $G\left(q_{m}\right) \subset G\left(q_{n}\right)$ and $F\left(q_{m}\right) \rho G\left(q_{n}\right)$ when $q_{m}<q_{n}$. Then there is a family $\left\{H\left(q_{n}\right)\right.$ : $\left.q_{n} \in Q\right\}$ such that $F\left(q_{m}\right) \rho H\left(q_{n}\right), H\left(q_{m}\right) \rho H\left(q_{n}\right)$ and $H\left(q_{m}\right) \rho G\left(q_{n}\right)$ when $q_{m}<q_{n}$.

2.6. THEOREM. In a bitopological space $(X, P, Q)$, the following are equivalent:

(1) $X$ is pairwise perfectly normal.

(2) Every $P(Q)-$-l.s.c. real function defined in $X$ is the limit of a monotonically increasing sequence of $P(Q)$-l.s.c. and $Q(P)$-u.s.c. functions.

(3) If $f$ is a real-valued function $P(Q)$-l.s.c. in $X$ and $Q(P)$-u.s.c. in the $Q(P)$-closed subset $C \subset X$, then $f$ is the limit of a monotonically increasing sequence $\left\{f_{n}\right\}_{n=1}^{\infty}$ of functions, $P(Q)-$ l.s.c. and $Q(P)$-u.s.c. in $X$ such that $f_{n}(x)=f(x)$ for every natural $n$ and every $x \in C$.

Proof. We prove the sequence $(1) \Rightarrow(3) \Rightarrow(2) \Rightarrow(1)$; moreover, we explain only the proofs for $P$ since the technique for $Q$ is the same.

$1 \Rightarrow 3$. Let $f$ be a real function defined in $X, P$-l.s.c. in $X$ and $Q$-u.s.c. in the $Q$-closed set $C \subset X$. For every $q_{n} \in Q$, let $F\left(q_{n}\right)=f^{-1}\left(\left(-\infty, q_{n}\right]\right)=\bigcap_{m=1}^{\infty} A_{n, m}$, where $A_{n, m}$ is $Q$-open, since $X$ is pairwise perfectly normal. It is no restriction to suppose $A_{n, m+1} \subset A_{n, m}$ for every $n, m$.

The sets $U_{n, m}=A_{n, m} \cup\left(\cup\left\{A_{s, m}: s<n\right.\right.$ and $\left.\left.q_{s}<q_{n}\right\}\right)$ have the properties of $A_{n, m}$ stated before and, furthermore, $U_{r, m} \subset U_{n, m}$ when $q_{r}<q_{n}$ and $r<n$. 
Now, let $V_{n, m}^{\prime}=U_{n, m} \cap\left(\cap\left\{U_{s, m}: s<n\right.\right.$ and $\left.\left.q_{s}>q_{n}\right\}\right)$; also $V_{n, m}^{\prime}$ is $Q$-open, $V_{n, m+1}^{\prime} \subset V_{n, m}^{\prime}$ for each $n, m$ and $F\left(q_{n}\right)=\bigcap_{m=1}^{\infty} V_{n, m}^{\prime}$. Moreover $V_{r, m}^{\prime} \subset V_{n, m}^{\prime}$ when $q_{r}<q_{n}$ : if $r<n, V_{r, m}^{\prime} \subset U_{r, m} \subset U_{n, m}$; therefore, when $s<n$ and $q_{s}>q_{n}$, if $r<s<n$ we have $V_{r, m}^{\prime} \subset U_{r, m} \subset U_{s, m}$ and if $s<r$ then $V_{r, m}^{\prime} \subset U_{s, m}$. On the other hand, if $r>n, V_{r, m}^{\prime} \subset U_{n, m}$, and therefore, when $s<n$ and $q_{s}>q_{n}, V_{r, m}^{\prime} \subset U_{s, m}$.

From $\left\{F\left(q_{n}\right)\right\}_{n=1}^{\infty}$ we can take a subsequence $\left\{F\left(q_{n_{i}}\right)\right\}_{i=1}^{\infty}$ such that $q_{n_{i+1}}<q_{n_{i}}$ for $i=1,2, \ldots$ and $\bigcap_{i=1}^{\infty} F\left(q_{n_{i}}\right)=\varnothing$. For every $q_{n} \in Q$, there is an $i$ such that $q_{n_{i}} \leqslant q_{n}<q_{n_{i-1}}$. Let $V_{n, m}=V_{n, i+m}^{\prime}$ for each natural $m$. The family $\left\{V_{n, m}\right\}_{m=1}^{\infty}$ has the same properties of $\left\{V_{n, m}^{\prime}\right\}_{m=1}^{\infty}$ stated before and verifies $\bigcap_{n=1}^{\infty} V_{n, m}=\varnothing$ for every natural $m$, because for each $x \in X$ there are numbers $r$ and $m_{0}$ such that $x \notin V_{r, m_{0}}^{\prime}$.

Finally, let $B_{n, m}=V_{n, m} \cap\left(f^{-1}\left(\left(-\infty, q_{n}\right)\right) \cup(X-C)\right)$, for every $n, m$. As $f$ is $Q$-u.s.c. in $C, B_{n, m}$ is $Q$-open. Also $B_{n, m+1} \subset B_{n, m}$, for every $n, m, B_{r, m} \subset B_{n, m}$ when $q_{r}<q_{n}$ and $\cap_{n=1}^{\infty} B_{n, m}=\varnothing$ for each $m$.

By taking $\mathscr{C}=P(X)$ and the relation $A \rho B$ if and only if $P$-cl $(A) \subset Q$-int $(B)$, the pairwise normality of $X$ allows us to apply Lemma 2.5 to the families $\left\{F\left(q_{n}\right)\right\}_{n=1}^{\infty}$ and $\left\{G_{m}\left(q_{n}\right)=B_{n, m}\right\}_{n=1}^{\infty}$ since, if $q_{r}<q_{n}, P-\operatorname{cl}\left(F\left(q_{r}\right)\right)=F\left(q_{r}\right) \subset$ $f^{-1}\left(\left(-\infty, q_{n}\right)\right) \subset \boldsymbol{F}\left(q_{n}\right) \cap\left(f^{-1}\left(\left(-\infty, q_{n}\right)\right) \cup(X-C)\right) \subset B_{n, m}=Q-\operatorname{int}\left(G_{m}\left(q_{n}\right)\right)$. This is true for every $m$, so by Lemma 2.5 , there are families $\left\{H_{m}\left(q_{n}\right)\right\}_{n=1}^{\infty}$, $m=1,2, \ldots$, such that $F\left(q_{r}\right) \rho H_{m}\left(q_{s}\right), H_{m}\left(q_{r}\right) \rho H_{m}\left(q_{s}\right)$ and $H_{m}\left(q_{r}\right) \rho G_{m}\left(q_{s}\right)$ when $q_{r}<q_{s}$, for $m=1,2, \ldots$

Since $\bigcap_{n=1}^{\infty} H_{m}\left(q_{n}\right)=\varnothing$ for every $m$, this family defines a real $P$-l.s.c. and $Q$-u.s.c. function $h_{m}$ on $X$ such that $h_{m} \leqslant f$ and $h_{m}(x)=f(x)$ for every $x \in C$. Then the family of functions $f_{n}=\max \left\{h_{m}\right\}_{m=1}^{n}$ is the desired monotonically increasing sequence of functions.

$3 \Rightarrow 2$. This is obvious with $C=\varnothing$.

$2 \Rightarrow 1$. Let $A$ be a $P$-closed subset of $X ; A$ is $G_{\partial}$ with respect to $Q$. The function

$$
f_{A}(x)= \begin{cases}1 & \text { when } x \in X-A \\ 0 & \text { when } x \in A\end{cases}
$$

is $P$-l.s.c. in $X$ and it is the limit of a monotonically increasing sequence $\left\{f_{n}\right\}_{n=1}^{\infty}$ of $P$-1.s.c. and $Q$-u.s.c. functions. It is immediate that $A=\bigcap_{n=1}^{\infty} f_{n}^{-1}((-\infty, 1 / n))$ and these sets are $Q$-open. Then $X$ is pairwise perfect.

Now let $A$ be a $P$-closed set and let $B$ be a $Q$-closed set disjoint from $A$. In the same way as in the previous paragraph, given $f_{A}$ and $f_{B}$, there are functions $\left\{f_{n}^{A}\right\}_{n=1}^{\infty}$ which are $P$-1.s.c. and $Q$-u.s.c. and $\left\{f_{n}^{B}\right\}_{n=1}^{\infty}$ which are $Q$-1.s.c. and $P$-u.s.c., for each $n$.

Let $h=\sum_{n=1}^{\infty} \frac{1}{2^{n}} \max \left(0, f_{n}^{A}\right)$ and let $k=\sum_{n=1}^{\infty} \frac{1}{2^{n}} \max \left(0, f_{n}^{B}\right)$; the set $(h-k)^{-1}((-\infty, 0))$ is a $Q$-neighbourhood of $A$ disjoint from the $P$-neighbourhood $(h-k)^{-1}((0, \infty))$ of $B$; therefore $X$ is pairwise normal. 


\section{Other properties}

In this section we study preservation of pairwise perfect normality by some kinds of functions, subspaces, sums and products.

We say that the function $f:\left(X, P_{1}, Q_{1}\right) \rightarrow\left(Y, P_{2}, Q_{2}\right)$ is continuous when $f$ : $\left(X, P_{1}\right) \rightarrow\left(Y, P_{2}\right)$ and $f:\left(X, Q_{1}\right) \rightarrow\left(Y, Q_{2}\right)$ are continuous. In an analogous way we define $f$ to be closed or perfect.

3.1. Proposition. Let $\left(X, P_{1}, Q_{1}\right)$ be a pairwise perfectly normal space and let $f$ : $\left(X, P_{1}, Q_{1}\right) \rightarrow\left(Y, P_{2}, Q_{2}\right)$ be a closed continuous function from $X$ onto $Y$. Then $\left(Y, P_{2}, Q_{2}\right)$ is pairwise perfectly normal.

Proof. The proof is easy by following the usual techniques of pairwise perfectly normal spaces.

3.2. COROLlaRY. The perfect image of a pairwise perfectly normal space is pairwise perfectly normal.

Now we study the pre-image of a pairwise perfectly normal space. Given a function $f: X \rightarrow(Y, T)$, we denote by $f^{-1}(T)=\left\{f^{-1}(A): A \in T\right\}$ the weak topology of $f$.

3.3. Proposition. Let $f: X \rightarrow(Y, U, V)$ be a function; let $P=f^{-1}(U)$ and $Q=f^{-1}(V)$. If $(Y, U, V)$ is pairwise perfectly normal then $(X, P, Q)$ is pairwise perfectly normal.

Proof. Given a $P$-closed set $A \subset X$, there is an $U$-closed set $A^{\prime} \subset Y$ such that $A=f^{-1}\left(A^{\prime}\right)$. Then, by 2.2 , there is an $U$-1.s.c. and $V$-u.s.c. function $g_{A}: Y \rightarrow[0,1]$ such that $g_{A}^{-1}(0)=A^{\prime}$. The function $f_{A}=g_{A} \cdot f$ is $P$-1.s.c. and $Q$-u.s.c. in $X$ and $f_{A}^{-1}(0)=f^{-1}\left(A^{\prime}\right)=A$.

Similarly we obtain the desired result from a $Q$-closed subset of $X$. Therefore, by $2.2,(X, P, Q)$ is pairwise perfectly normal.

Corollary. Let $(X, P, Q)$ be pairwise perfectly normal and let $Y \subset X$. Then the bitopological subspace $Y$ is pairwise perfectly normal.

Proof. The inclusion $i: Y \rightarrow(X, P, Q)$ satisfies $\left.P\right|_{Y}=i^{-1}(P)$ and $\left.Q\right|_{Y}=$ $i^{-1}(Q)$. 
With respect to the preservation of the pairwise perfect normality by products or quotients, there are examples proving that this is not true even for perfectly normal topological spaces.

3.5. EXAMPLes. (1) Let $I=[0,1]$ with its usual topology. This space is perfectly normal, but $I^{I}$ is not perfectly normal because $(0,1)^{I}$ is not normal and then $I^{I}$ is not completely normal.

(2) Perfect normality is not preserved by finite products as is shown by the space $(R, T)$, where $T$ is the topology whose base is $\{[a, b): a, b \in \mathbf{R}\}$.

(3) The usual real topological space $(\mathbf{R}, U)$ is perfectly normal but its quotient $(\mathbf{R} / \mathbf{Q}, U / \mathbf{Q})$, where $\mathbf{Q}$ is the rational set, is not perfectly normal.

3.6. Proposition. Let $\left\{\left(X_{i}, P_{i}, Q_{i}\right), i \in I\right\}$ be a family of bitopological spaces such that their bitopological product $\left(\Pi X_{i}, P, Q\right)$ is pairwise perfectly normal. Then every factor is pairwise perfectly normal.

Proof. Let $A$ be a $P_{i}$-closed subset of $X_{i}$. Then $A \times \Pi\left\{X_{j}: j \neq i\right\}$ is $P$-closed and therefore $A \times \Pi\left\{X_{j}: j \neq 1\right\}=\bigcap_{n=1}^{\infty} A_{n}$, where each $A_{n}$ is $Q$-open. Then $A=\bigcap_{n=1}^{\infty} \Pi_{i}\left(A_{n}\right)$, where each $\Pi_{i}\left(A_{n}\right)$ is $Q_{i}$-open.

By following the same technique, we prove that $X_{i}$ is pairwise normal.

3.7. THEOREM. The bitopological sum $\left(\sum_{i \in I} X_{i}, P, Q\right)$ is pairwise perfectly normal if and only if each space $\left(X_{i}, P_{i}, Q_{i}\right)$ is pairwise perfectly normal.

Proof. The necessity follows from 3.4 and the sufficiency is immediate.

\section{References}

[1] S. P. Arya and A. Singhal, "A note on pairwise $D_{1}$ spaces", Glasnik Math. 14 (34) (1979), 147-150.

[2] J. G. Ceder, "Some generalisations of metric spaces", Pacific J. Math. 11 (1961), 105-125.

[3] A. Gutiérrez and S. Romaguera, "Sobre espacios pairwise estratificables", Rev. Roumaine Math. Pures Appl., to appear.

[4] E. P. Lane, "Bitopological spaces and quasi-uniform spaces”, Proc. London Math. Soc. (3) 17 (1967), 241-256.

[5] M. López and J. L. Santos, "A necessary and sufficient condition for the insertion of a continuous function", Proc. Amer. Math. Soc., to appear.

[6] C. W. Patty, “Bitopological spaces”, Duke Math. J. 34 (1967), 387-392. 
[7] S. Romaguera, "Two characterizations of quasi-pseudometrizable bitopological spaces", $J$. Austral. Math. Soc. Ser. A 35 (1983), 327-333.

[8] H. Tong, "Some characterizations of normal and perfectly normal spaces", Duke Math. J. 19 (1952), 289-292.

Cátedra de Matemáticas

E.T.S.I. Agrónomos

Universidad Politécnica

Camino de Vera s.n.

46022-Valencia

Spain
Departamento de Matemáticas

E.U. del Profesorado de E.G.B.

Alcalde Reig, 8

46006-Valencia

Spain 